\title{
APPLICATION OF TRIPLE CORRELATION AND BISPECTRUM FOR INTERFERENCE IMMUNITY IMPROVEMENT IN TELECOMMUNICATIONS SYSTEMS
}

\author{
Pavel Molchanov*, Alexandr Totsky ** \\ * National Aerospace University \\ Chkalova Str. 17, 61070, Kharkov, Ukraine \\ e-mail:avm@datasvit.net \\ ** Department of Transmitters, Receivers and Signal Processing \\ National Aerospace University, Chkalova Str. 17, 61070, Kharkov, Ukraine \\ e-mail: totskiy@xai.edu.ua
}

\begin{abstract}
This paper presents a new noise immunity encoding/decoding technique by using the features of triple correlation and bispectrum widely employed in digital signal processing systems operating in noise environments. The triple correlationand bispectrum-based encoding/decoding algorithm is tested for a digital radio telecommunications binary frequency shift keying system. The errorless decoding probability was analyzed by means of computer simulation for the transmission and reception of a test message in a radio channel disturbed by both additive white Gaussian noise (AWGN) and a mixture of an AWGN and an impulsive noise. Computer simulation results obtained for varying and less than unity signal-to-noise ratios at the demodulator input demonstrate a considerable improvement in the noise immunity of the technique suggested in comparison with the traditional redundant linear block encoding/decoding technique.
\end{abstract}

Keywords: telecommunications, frequency shift keying, redundant encoding, triple correlation, bispectrum.

\section{Introduction}

In many real-life situations, mobile communication multipath radio channels exhibit the influence of fading and power electrical interferences. The latter may provoke intolerable errors in digital radio communication systems operating under low and time-varying signal-to-noise ratios (SNRs). Hence, to provide a desirable communication reliability, it is necessary to select an adequate noise immunity encoding/decoding technique. However, in the case when the interference varies within a wide dynamic range during a communication session, it is problematic to find a reliable encoding technique for providing the required noise immunity and satisfactory message errors.

The bit error probability $p_{e}$ assessed at the output of the correlation detection scheme according to the maximum-likelihood decoding rule is given by the following well-known condition (Sklar, 2003):

$$
p_{e} \leq \frac{M}{2} Q\left(\sqrt{\frac{E_{w}(1-\rho)}{N_{0}}}\right)
$$

$$
=\frac{M}{2} Q\left(\sqrt{\frac{P_{w}(1-\rho)}{\sigma^{2}}} B\right),
$$

where $Q(x)$ is the Gaussian error integral, $M=2^{k}$ is the coder alphabet capacity, $\kappa$ is the number of information bits per code word, $E_{w}=k E_{b}, P_{w}=k P_{b}, E_{b}$ and $P_{b}$ are the binary signal energy and power, respectively, $N_{0}$ and $\sigma^{2}$ are the AWGN spectral density and variance, respectively, $B=F k T_{b}$ is the signal base, $F$ is the signal spectrum bandwidth, $T_{b}$ is the time interval fixed for the transmission of binary symbols, $\rho$ is the cross-correlation coefficient of the binary signals $s_{1}(t)$ and $s_{2}(t)$ which is

$$
\rho=\frac{1}{E_{b}} \int_{0}^{T_{b}} s_{1}(t) s_{2}(t) \mathrm{d} t .
$$

According to (1), the required noise immunity can be achieved in various ways. One of them employs noise-like signals having large signal base $B$ values (Varakin, 1985). However, using wideband signals inevitably requires wi- 
dening the telecommunications system bandwidth. Unfortunately, the latter requirement is difficult or impossible to implement in practice due to the known limitations imposed on the bandwidth value. Moreover, the signal duration $k T_{b}$ must be as short as possible to obtain a desirable transmission rate that is conventionally limited.

One more noise immunity technique widely used in digital radio telecommunications systems is block coding (Sklar, 2003; Proakis, 1989), based on the insertion of redundancy in the information bit stream. According to the block coding approach, the bit stream is grouped in the sequence of words (blocks). The length of each block is assumed to be equal to $k\left(M=2^{k}\right)$. As a result, the bit error probability $p_{e}$ can be decreased without increasing the binary signal power $P_{b}$ in (1).

However, the block coding technique requires the widening of the bandwidth for preserving the desirable transmission rate. In the case when increasing the bandwidth is impossible, the time delay must be increased to provide the required value of the error probability $p_{e}$.

$>$ From linear block coding theory and maximumlikelihood decoding (Proakis, 1989) it follows that the correction of the error $e$ is possible in the case when the Hamming distance $d_{H}$ between two arbitrary words from the selected code word set satisfies

$$
d_{H} \geq 2 e+1 .
$$

Note that the condition (2) requires memorizing and storing the total number of code words equal to $2^{k}$. It is well known that the noise immunity increases in parallel to the increasing of the block length. However, the procedure of multiple correlation comparison of a code word received with a large total number of the code words requires a sufficient processing time and its efficiency decreases with the reduction in the SNR at the communication system input.

Thus the noise immunity of the digital radio telecommunications system can be achieved by:

- using binary orthogonal signals,

- increasing the signal base,

- introducing redundancy for error detection and correction.

Searching for improving the digital radio telecommunications system performance in the sense of the enhancement of its noise immunity lead us to the idea of constructing a new redundant encoding/decoding scheme by using the interference protection properties of triple correlation and bispectrum.

Our strategy is based on the application of such properties of triple correlation and bispectrum that are widely employed in digital signal processing for solving problems of the detection and recovery of non-Gaussian processes embedded in additive Gaussian noise for radar sys- tems (Totsky et al., 2003), bispectrum-based signal reconstruction in noisy environments (Totsky et al., 2006), biomedical engineering (Nakamura, 1993), and many other applications (Nikias and Raghuveer, 1987).

The goal of this paper is to develop a new noise immunity encoding/decoding technique based on using the code words constructed by quantized samples of triple correlation for two a-priori inserted subsidiary digital sequences. To recover a binary symbol, we propose to employ the maximum of the bispectrum magnitude (bimagnitude) of the above-mentioned subsidiary digital sequences as a test statistic. The bimagnitude is a robust estimate with respect to additive interference and it is insensitive to translations of the binary code sequence received.

The remainder of this paper is organized in the following manner: In Section 2, the proposed encoding and decoding algorithms are described. The approach suggested is tested by computer simulation of the transmission and reception of a binary coded test massage in the communication channel corrupted by AWGN and a mixture of AWGN and impulsive noise. Computer simulation results and a comparative analysis of the errorless probabilities calculated for the proposed coding technique and a conventional linear repetition code are presented and discussed in Section 3. Finally, the last section contains some concluding remarks.

\section{Proposed encoding / decoding algorithms}

2.1. Encoding algorithm using triple correlation function samples. A hallmark of the proposed approach is a two-stage encoding procedure. First, two orthogonal subsidiary code sequences are generated. Second, two redundant code sequences are generated in the form of samples of triple correlation functions (TCFs) of these subsidiary code sequences. Below a number of the proposed encoding procedures are enumerated.

Procedure 1. Two arbitrary selected orthogonal subsidiary eight-element code sequences, e.g., $\mathbf{a}=(00000000)$ and $\mathbf{b}=(22222221)$ are generated. The sequence $\mathbf{a}$ is assumed to correspond to the logic "zero" and the sequence $\mathbf{b}$ corresponds to the logic "unit" in the original binary message (the original information bit stream).

Note that while increasing the mentioned subsidiary code length (more than 8 elements considered in our illustrative example), the noise immunity will increase. However, at the same time, the message time delay will increase.

Procedure 2. Compute TCFs $R_{a}(l, m)$ and $R_{b}(l, m)$ of the above-mentioned code sequences $\mathbf{a}=(00000000)$ 


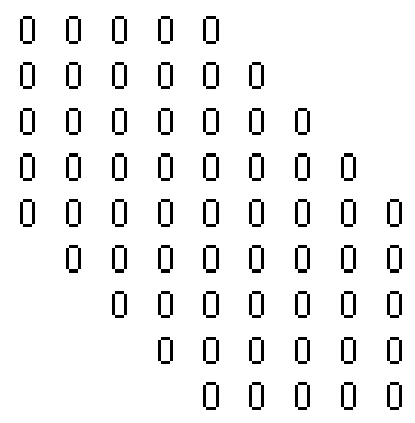

(a)

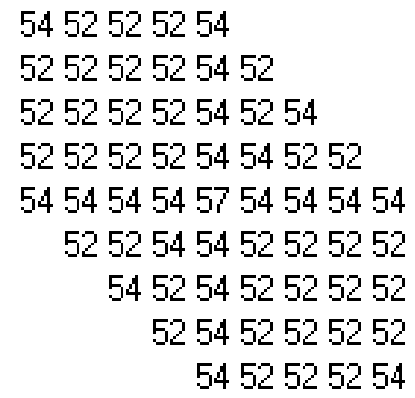

(b)

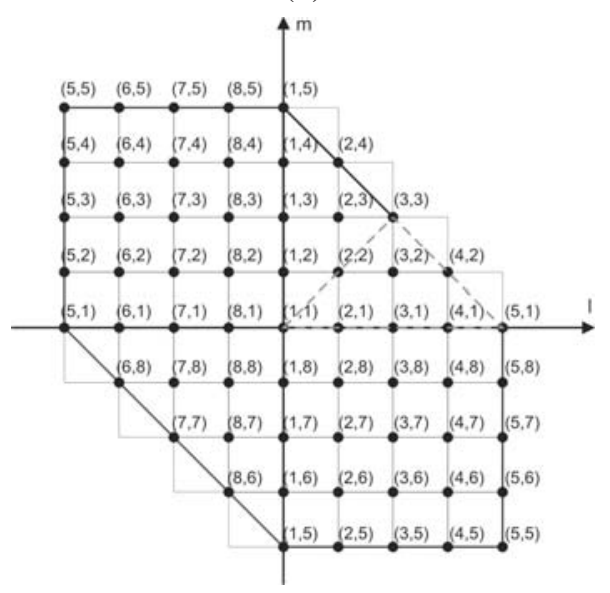

(c)

Fig. 1. TCFs $R_{a}(l, m)$ and $R_{b}(l, m)$ for the sequences $a$ (a) and $b$ (b), and the sample labelling introduced in the TCFs (c).

and $\mathbf{b}=(2222221)$ that are defined as

$$
\begin{aligned}
& R_{a}(l, m)=\sum_{n=1}^{8} a(n) a(n+l-1) a(n+m-1), \\
& R_{b}(l, m)=\sum_{n=1}^{8} b(n) b(n+l-1) b(n+m-1),
\end{aligned}
$$

where $l=1,2, \ldots, 8$ and $m=1,2, \ldots, 8$ are the translation indices. Note that the indices $l, m$, and $n$ in the formulas (3a) and (3b) are of only positive values.

The distributions of the TCF samples computed for the sequences $\mathbf{a}$ and $\mathbf{b}$ considered are shown in Fig. 1.
The analysis of the data represented in Fig. 1 reveals the following well-known peculiarities of TCFs (Nikias and Raghuveer, 1987). The TCF samples are distributed in the limits of a typical hexagon in the $(l, m)$ plane. Since the positive translation indices $l$ and $m$ considered are equal to even values of $L=M=8$, the symmetry of the hexagon with respect to the origin sample $R(1,1)$ in Fig. 1 is illustrated using the additional 9-th row and line due to the known TCF symmetry and periodicity properties. This TCF symmetry feature permits to compute TCFs only in the limits of the main triangular domain shown in Fig. 1(c). Note that such 12 triangular domains form the hexagon in Fig. 1. The latter peculiarity will be employed below for the construction of a proposed redundant code.

Procedure 3. Transform the decimal samples (3a) and (3b) to binary $r$-bit values ( $r=6$ for the illustrative example considered). As a result, redundant binary code arrays $\mathbf{C}(l, m)$ and $\mathbf{D}(l, m)$ are obtained and prepared for the transmission of a binary message.

Let us represent both the code arrays $\mathbf{C}(l, m)$ and $\mathbf{D}(l, m)$ by a code sequence containing eight-element lines according to their locations, cf. Fig. 1. We define the ordering in the code sequence in the following way. The first line in our code includes the samples belonging to the central horizontal line in Fig. 1. Note that the first code word in our code arrays corresponds to the maximum value of the TCF. As a result, at the coder output we obtain the following code arrays prepared for transmission in the radio channel:

$$
\begin{gathered}
C(l, m)=\left(\begin{array}{l}
R_{a}(1,1), \ldots, R_{a}(8,1) \\
R_{a}(1,2), \ldots, R_{a}(8,2) \\
\ldots \ldots \ldots \ldots \ldots \ldots \ldots \ldots . \\
R_{a}(1,8), \ldots, R_{a}(8,8)
\end{array}\right), \\
D(l, m)=\left(\begin{array}{l}
R_{b}(1,1), \ldots, R_{b}(8,1) \\
R_{b}(1,2), \ldots, R_{b}(8,2) \\
\ldots \ldots \ldots \ldots \ldots \ldots \ldots \ldots \\
R_{b}(1,8), \ldots, R_{b}(8,8)
\end{array}\right) .
\end{gathered}
$$

The maximum number of words in the redundant codes (4a) and (4b) equals 64 elements.

Procedure 4. The TCF symmetry property (Nikias and Raghuveer, 1987)

$$
\begin{aligned}
R(l, m) & =R(m, l)=R(l-m,-m) \\
& =R(m-l,-l)=R(-l, l-m)
\end{aligned}
$$

implies that the TCF (3b) exhibits proper redundancy, i.e., only three dissimilar values, namely, 57,54 and 52 belong to the function $R_{b}(l, m)$ (3b) in the main triangular region (see Fig. 1(c)). These values are repeated several times in the hexagonal domain $(l, m)$ due to the above-mentioned TCF symmetry property. It should be noted that for the 
discussed case of positive indices, we use only the first equality in (5).

It is important to note that these three values unambiguously define the TCF $R_{b}(l, m)$. Therefore, the number of code words can be sufficiently shortened in the truncated code array without any loss of the information transmitted.

Taking into account the symmetry property (5), new short (truncated) code sequences can be constructed in the form of a set containing only the following three six-code code words:

$$
\begin{aligned}
& \mathbf{C}_{T}(l, m)=R_{a}(1,1) ; R_{a}(1,2) ; R_{a}(2,3), \\
& \mathbf{D}_{T}(l, m)=R_{b}(1,1) ; R_{b}(1,2) ; R_{b}(2,3) .
\end{aligned}
$$

Since the number of code words in $(6 a)$ and $(6 b)$ is sufficiently smaller when compared with (4a) and (4b), the transmission and decoding performed for truncated codes will take sufficiently shorter time intervals.

\subsection{Demodulation and decoding procedures sugge-}

sted. As a real-life example, we consider a digital radio telecommunications system with binary frequency shift keying (FSK) manipulation. Although many other manipulation systems are conventionally in use, the FSK manipulation is a common one. Orthogonal FSK signals $s_{1}(t)$ (logical "zero") and $s_{2}(t)$ (logical "unit") transmitted during the limited bit time interval $\left[0, T_{b}\right]$ can be written as

$$
\begin{array}{ll}
s_{1}(t)=A_{s} \cos \left(2 \pi f_{1} t\right), & T_{b} \geq t \geq 0, \\
s_{2}(t)=A_{s} \cos \left(2 \pi f_{2} t\right), & T_{b} \geq t \geq 0 .
\end{array}
$$

Let us assume that the received waveform $r(t)$ obtained at the FSK demodulator output is corrupted by an additive interference as

$$
r(t)=s_{i}(t)+n_{G}(t)+n_{p}(t), \quad i=1,2,
$$

where $n_{G}(t)$ is AWGN whose spectral power density and variance are equal to $N_{0}$ and $\sigma^{2}$, respectively, $n_{p}(t)$ being impulsive noise.

A remarkable feature of the proposed approach is the two-stage recovery of binary symbols in a decoding device.

First, a standard correlation detection procedure of the binary FSK signals corrupted by additive noise (8) is performed with the maximum-likelihood method. The noise-free signals (7a) and (7b) serve as the references in a coherent correlation demodulator.

As a result, the estimates of binary six-bit code words $\hat{C}(l, m)$ and $\hat{D}(l, m)$ (or truncated ones $\hat{C}_{\tau}(l, m)$ and $\left.\hat{D}_{\tau}(l, m)\right)$ are recovered at the demodulator output. Note that part of the symbols in the information bit stream received may be renamed, i.e., a message may be corrupted by the interferences $n_{G}(t)$ and $n_{p}(t)$.
It should be stressed that the same 12 triangular domains exist in the total TCF hexagon domain due to the above-mentioned symmetry property (5) (see Fig. 1). This important feacture allows us to average the TCF samples $\hat{C}(l, m)$ and $\hat{D}(l, m)$ belonging to the corresponding twelve triangular domains. Therefore, the noise immunity for the codes $\hat{C}(l, m)$ and $\hat{D}(l, m)$ can be sufficiently improved by using this average procedure when compared with the truncated codes $\hat{C}_{\tau}(l, m)$ and $\hat{D}_{\tau}(l, m)$.

Second, the following bispectrum estimates performed in the decoding device are determined:

$$
\begin{aligned}
\hat{B}_{a}(p, q) & =\operatorname{FFT}\left[\hat{R}_{a}(l, m)\right], \\
\hat{B}_{b}(p, q) & =\operatorname{FFT}\left[\hat{R}_{b}(l, m)\right],
\end{aligned}
$$

where $p=1,2, \ldots, 8$ and $q=1,2, \ldots, 8$ are the indices of the independent frequency samples, FFT denotes a fast Fourier transform, $\hat{R}_{a}(l, m)$ and $\hat{R}_{b}(l, m)$ are the TCF estimates recovered from the code sequences $\hat{C}(l, m)$ and $\hat{D}(l, m)$ (or the truncated code sequences $\hat{C}_{T}(l, m)$ and $\hat{D}_{T}(l, m)$, respectively).

The module values of the bispectrum estimates (9a) and $(9 b)$ called "bimagnitudes" serve as test statistics for the recovery of the subsidiary code sequences $\mathbf{a}$ and $\mathbf{b}$ generated in the coder.

A maximum-likelihood detector makes a twoalternative decision according to hypotheses $H_{a}$ and $H_{b}$ as

$$
\max \{|\hat{B}(p, q)|\}=\left\{\begin{array}{l}
>\gamma \Rightarrow H_{b}, \\
<\gamma \Rightarrow H_{a},
\end{array}\right.
$$

where $\gamma$ is a threshold value given a priori in the decision device. Note that a proper choice of the subsidiary code sequences $a$ and $b$ will make it possible to obtain a robust noise protected bimagnitude statistic $\max \{|\hat{B}(p, q)|\}$ for a concrete noise environment.

After making a decision according to the criterion (10), the received message is recovered at the decoder output.

\section{Discussion of computer simulation results}

In this section the performance of the proposed encoding/decoding technique will be studied, discussed and compared with a conventional linear repetition block code $(6,1)$. We chose this repetition block code for two reasons. First, in practice this repetition block coding technique exhibits perfect effectiveness in a mobile radio communication system where the mobile unit travels at rapidly changing speeds and, hence, the SNR may vary within wide limits. Second, for an adequate comparative analysis, we have to select the same code parameters, namely, the dimension of the repetition code and the length of the code word in the proposed coding technique.

As a typical practical example, we will consider the original message given in the ANSI alphabet. The binary 


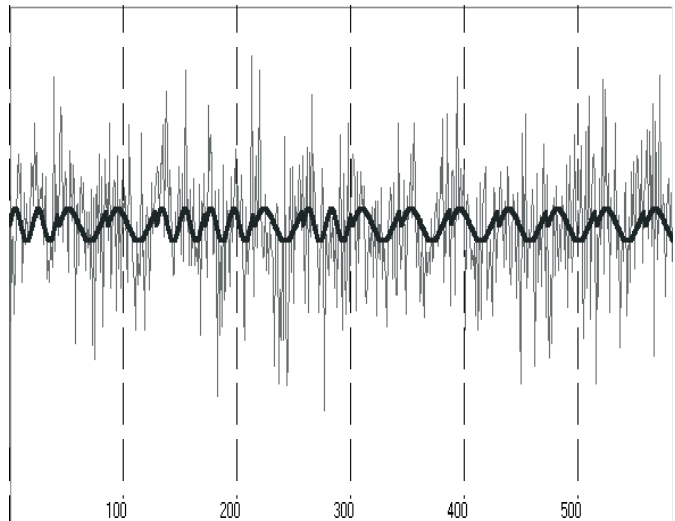

Fig. 2. Segment of the FSK signal with amplitude $A_{s}=10 \mathrm{mV}$ corrupted by AWGN with variance $\sigma^{2}=1000 \mathrm{mV}$ (the thick curve corresponds to the original binary FSK signals).

FSK signals (7a) and (7b) with the tone values of $f_{1}=$ $1200 \mathrm{~Hz}$ and $f_{2}=2200 \mathrm{~Hz}$ as well as the binary signal duration of $T_{b}=1 /\left(f_{2}-f_{1}\right)$ were used in our computer simulations.

The estimation of the noise immunity of the proposed technique was performed by an analysis of the errorless decoding probability. This probability was computed in the form of an averaged number of errorless symbols decoded with respect to their total number belonging to the original message.

The radio communication channel was modelled as a memoryless channel. The errorless decoding probability was computed depending on the binary signal amplitude $A_{s}$ given in (7a) and (7b) as well as fixed AWGN variance. The signal amplitude $A_{s}$ was varied within the limits of $[3,35] \mathrm{mV}$. It permits us to vary the SNR in a wide range.

Note that the varying limits of $A_{s}$ considered correspond to the changing of power SNR $=P_{b} / \sigma^{2}$ at the FSK demodulator input from 0.0045 up to 0.61 for the fixed AWGN variance equal to $\sigma^{2}=1000 \mathrm{~mW}$. It should be stressed that such SNR variation can be caused in practice by a multi-path fading channel in mobile telecommunications systems. The bimagnitude threshold in the decision rule (10) was selected to be equal to $\gamma=1529$.

According to the above-mentioned symmetry property of the TCF, the bit sequences generated by only nine code words, namely, by one word $R_{a}(1,1)$ (or $R_{b}(1,1)$ ) corresponding to the maximum TCF value and fourfold repeated code words $R_{a}(1,2)$ and $R_{a}(2,3)$ (or words $R_{b}(1,2)$ and $R_{b}(2,3)$ ) were used for the transmission of the test message for the truncated codes (6a) and (6b).

The curves of segment realizations of the FSK signals corrupted by AWGN and a mixture of AWGN and impulsive noise are shown in Figs. 2 and 3, respectively.

The graphs of errorless decoding probabilities computed for the code sequences (4a), (4b), (6a) and (6b)

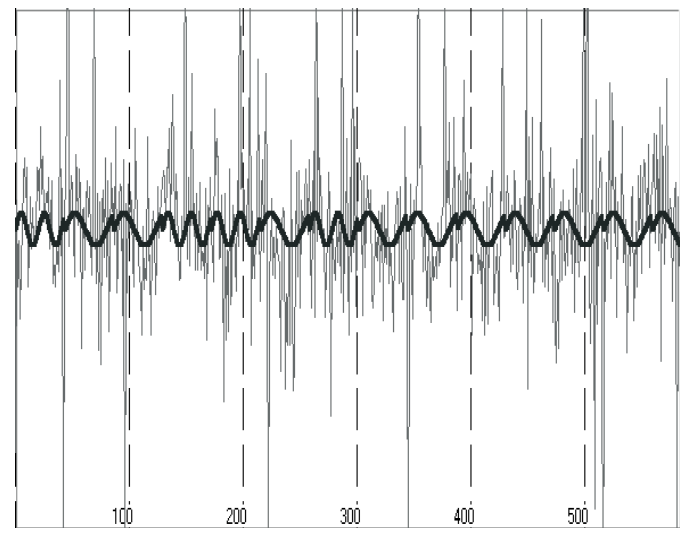

Fig. 3. Segment of the FSK signal with amplitude $A_{s}=10 \mathrm{mV}$ corrupted by a mixture of AWGN with variance $\sigma^{2}=$ $1000 \mathrm{mV}$ and impulsive noise with spike amplitude $A_{p}=200 \mathrm{mV}$ and the probability of negative and positive pulses equal to $p=5 \%$.

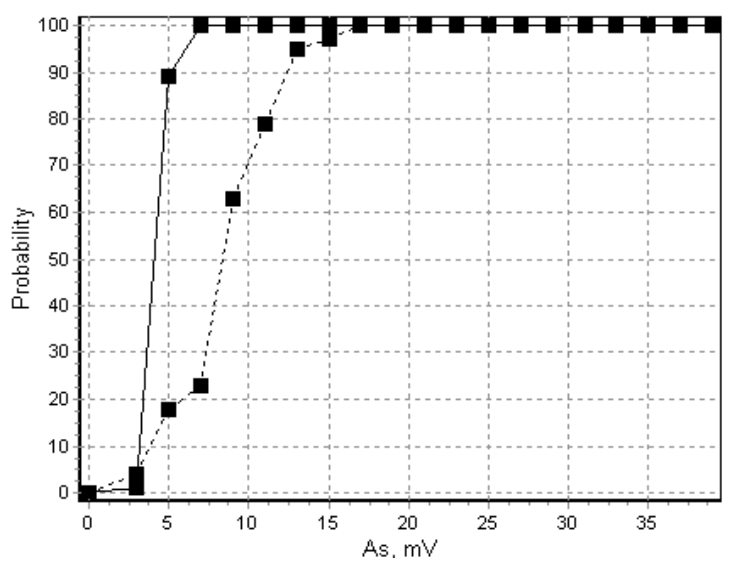

Fig. 4. Errorless decoding probability as a function of the binary FSK signal amplitude obtained for the code sequences (4a) and (4b) in a radio channel corrupted by AWGN.

for the test message "xai504" transmitted over a radio channel corrupted by AWGN with fixed noise variance of $\sigma^{2}=1000 \mathrm{mV}$ are shown in Figs. 4 and 5, respectively.

The graphs of the errorless decoding probabilities computed for the code sequences (4a), (4b), (6a) and (6b) for a test message "xai504" in a radio channel corrupted by a mixture of AWGN $\sigma^{2}=100 \mathrm{mV}$ and impulsive noise (the spike amplitude is equal to $A_{p}=200 \mathrm{mV}$ and the probability of negative and positive pulses is equal to $p=5 \%$ ) are shown in Figs. 6 and 7, respectively.

The results demonstrated in Figs. 4-7 were computed for 30 Monte Carlo runs, i.e., by using 30 independent radio telecommunications sessions for the same test message in the noisy memoryless channel. The curves in the graphs computed for the code $(6,1)$ are marked by dotted lines and the results obtained for the proposed code are represented by solid curves.

$>$ From Figs. 4 and 5 it is clearly seen that the binary 


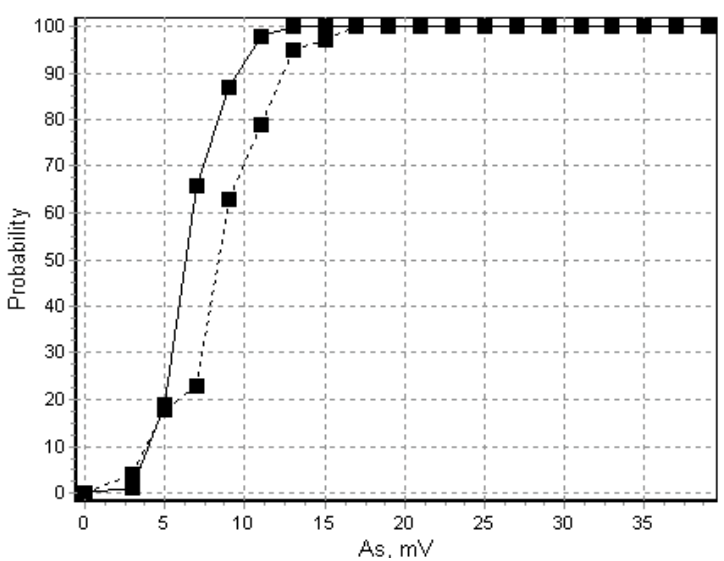

Fig. 5. Errorless decoding probability as a function of the binary signal amplitude obtained for the truncated code sequences $(6 a)$ and $(6 b)$ in a radio channel corrupted by AWGN.

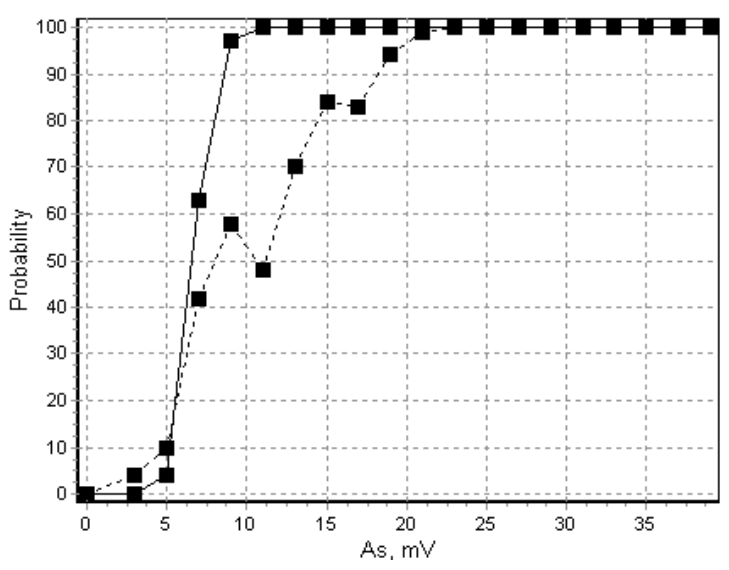

Fig. 6. Errorless decoding probability as a function of the binary signal amplitude obtained for the code sequences (4a) and (4b) in a radio channel corrupted by a mixture of AWGN and impulsive noise.

signal threshold amplitude providing errorless decoding for the block coding $(6,1)$ is equal to $A_{T}=18 \mathrm{mV}$ (the corresponding threshold SNR is approximately equal to $\left.\left(P_{b} / \sigma^{2}\right)_{T}=0.16\right)$. The corresponding binary signal threshold amplitudes obtained for the proposed codes (4a), (4b), (6a) and (6b) are equal to $A_{T}=7 \mathrm{mV}\left(\left(P_{b} / \sigma^{2}\right)_{T}=\right.$ $0.0245)$ and $A_{T}=12 \mathrm{mV}\left(\left(\left(P_{b} / \sigma^{2}\right)_{T}=0.072\right)\right.$, respectively. Therefore, the benefit assessed for the proposed code in comparison with the block code $(6,1)$ by the corresponding ratios $\left(P_{b} / \sigma^{2}\right)_{T}$ is equal to 6.5 times $(8.1 \mathrm{~dB})$ for the code (4a), (4b) and 2.2 times $(3.4 \mathrm{~dB})$ for the code (6a), (6b), respectively.

The comparative analysis of the noise immunity of the proposed TCF coding and the conventional code $(6,1)$ with respect to the practically important case of the influence of impulsive noise (see Figs. 6 and 7) also demonstrates good robustness of the proposed codes. From the

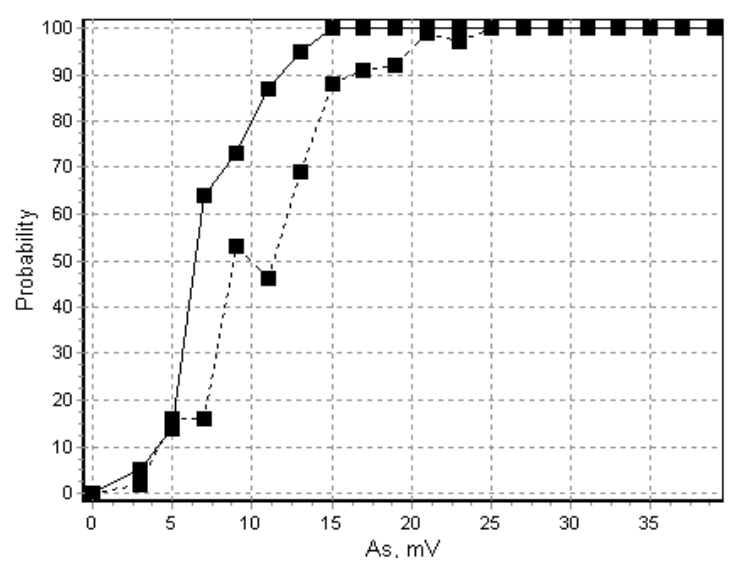

Fig. 7. Errorless decoding probability as a function of the binary signal amplitude obtained for the code sequences (6a) and (6b) in a radio channel corrupted by a mixture of AWGN and impulsive noise.

plots it follows that the conventional code $(6,1)$ provides errorless decoding with a binary signal threshold amplitude equal to $A_{T}=22 \mathrm{mV}$. The proposed codes (4a), (4b) and (6a), (6b) provide errorless decoding in a mixed noise environment for $A_{T}=11 \mathrm{mV}$ and $A_{T}=15 \mathrm{mV}$, respectively. The better performance of the proposed TCF coding technique is clearly evidenced in the curves plotted in Figs. 6 and 7.

\section{Concluding remarks}

A novel encoding/decoding technique to improve the noise immunity in digital radio telecommunications systems was proposed and tested via computer simulations. The capacity of the proposed redundant code alphabet can be selected in the form corresponding to the total or truncated code sequence depending on the trade-off existing obviously between the SNR value and required message transmission and decoding rates.

The proposed technique makes it possible to improve the reliability of digital radio communication systems operating under low (less than unity) and varying SNRs. Unfortunately, the message time delay in the proposed coding technique increases due to the insertion of a redundant code. However, we suppose that our coding technique may be useful in a situation when errorless transmission is of greatest importance, and the time delay is a less important parameter in a radio communication system.

The results of this paper will be of use in digital mobile communication systems operating under low and variable SNRs as well as with messages transmitted over radio channels corrupted by a mixture of AWGN and impulsive noise signals. 


\section{References}

Nakamura M. (1993). Waveform estimation from noisy signals with variable signal delay using bispectrum averaging, IEEE Transactions on Biomedical Engineering 40(2): 118-127.

Nikias C. L. and Raghuveer M. R. (1987). Bispectral estimation: A digital signal processing framework, Proceedings of the IEEE 75(7): 869-891.

Proakis J. G. (1989). Digital Communications, 2nd Edn., McGraw-Hill, New York, NY.

Sklar B. (2003). Digital Communication: Fundamentals and Applications, 2nd Edn., Williams Publishing House, Moscow, (in Russian).

Totsky A. V., Kurbatov I. V., Lukin V. V., Egiazarian K. O., and Astola J. T. (2003). Combined bispectrum-filtering techniques for radar output signal reconstruction in ATR applications, Proceedings of the International Conference on Automatic Target Recognition XIII, Orlando, FL, USA, The International Society for Optical Engineering, Vol. 5094, pp. 301-312.
Totsky A. V., Fevralev D. V., Lukin V. V., Katkovnik V. Ya., Paly D. V., Egiazarian K. O., Pogrebnyak O. B. and Astola J. T. (2006). Performance study of adaptive filtering in bispectral signal reconstruction. Circuits, Systems, and Signal Processing 25(3): 314-342.

Varakin L. E. (1985). Communication Systems Using Noise-Like Signals, Radio and Svyaz, Moscow, (in Russsian).

Received: 5 January 2007

Revised: 20 March 2007

Re-revised: 10 May 2008 
